\title{
Camera calibration: a personal retrospective
}

\author{
Zhengyou Zhang ${ }^{1}$
}

Published online: 29 September 2016

(C) Springer-Verlag Berlin Heidelberg 2016

\begin{abstract}
The following article is the first in a new series of retrospective essays that will be published in Machine Vision and Applications. Unlike regular articles, retrospectives are short invited articles by distinguished researchers that outline the key ideas behind a wellknown piece of computer vision research as well as briefly describe the history of its invention and development from a personal perspective.

In the first article of this series, Dr. Zhengyou Zhang presents a retrospective on Zhang's method, which enables any user to quickly calibrate a camera from just a few views of a planar pattern. Since its introduction in 1999, it quickly became the standard technique for camera calibration both in the computer vision community and beyond.
\end{abstract}

- Rahul Sukthankar, Editor in Chief

Computer vision is a key branch of artificial intelligence, aiming at understanding the surrounding environment from the visual information captured by cameras. 3D computer vision is a subfield of computer vision that focuses on extracting 3D metric information from 2D images. This is a very challenging task since what we have in an image is the projection of a 3D scene, whose depth information is lost during image formation (image projection). However, it has many important applications such as the following:

- Robot/Car/Drone/Visually impaired Navigation. 3D information is needed in this application for obstacle

\footnotetext{
Zhengyou Zhang

zhang@microsoft.com

1 Microsoft Research, Redmond, WA, USA
}

avoidance, free space determination, and pose estimation.

- Virtual/Augmented Reality (VR/AR). 3D computer vision is crucial for VR/AR. This includes camera pose estimation for head-mounted displays, 3D modeling for VR/AR contents, and seamless integration of virtual objects into the real world.

- Human-Computer/Robot Interaction. 3D computer vision allows the computer/robot to see the user, understand the user's nonverbal body language (gesture, body posture, facial expression, etc.), and interact in natural ways such as gesture and speech, instead of the traditional keyboard and mouse.

When I joined Microsoft Research early 1998, Microsoft's mission was to put a computer on every desk and in every home; my mission was to empower every computer with the visual capability of a human. I created a vision document called "Desktop Vision System" (DVS), with "vision" implying both "computer vision" and my vision for the future of desktop computers. In my DVS document, I set up my agenda along three lines of research:

- 3D object modeling. I imagined that products such as furniture would all have 3D photorealistic models. Before I bought them, I would use the DVS to build a 3D model of my house, import the furniture model, conduct lighting simulations, and determine whether it fits the house and where it fits the best.

- 3D face modeling. I imagined the DVS would create a 3D digital replica of each user, and this digital avatar would be a faithful representation of the user in 3D geometry, appearance, and dynamics, to be used when playing online games with others or when interacting with others in an online world. 
- Human emotion understanding. I imagined the DVS would understand the user's facial expression, and, depending on the user's mood, the computer would react differently while interacting with the user. For example, when the user approached, the DVS would recognize that the user is not in a happy mood; it would then play soothing music, and chat with sympathy to improve the user's mood.

I have carried out all three lines of research to certain degrees of success. An early piece of work on 3D object modeling appeared at ICCV 2001 [2]. A live demo on 3D face modeling was shown at Bill Gates' keynote in launching Xbox in 2001, and the work was summarized in a book published by the Cambridge University Press in 2011 [1]. The emotion-understanding work was shipped as part of Microsoft Cognitive Services (https://www.microsoft.com/ cognitive-services/en-us/emotion-api), and an initial work was published in the IJPRAI journal [4].

Having presented the context, I will now describe my invention on camera calibration, now known as Zhang's method. In order to fulfill the tasks that we described above with desired performance, the DVS needs to know the characteristics of its visual sensor (camera). That is the task of camera calibration, which is a process of determining the camera's internal parameters (focal length, aspect ratio and rectangularity of a pixel, the center of the image sensor) and the camera's external parameters (position and orientation). And that was my first project after joining Microsoft Research, working toward my Desktop Vision System vision.

Before I attacked the problem, there was already a technique available, which consisted of using a precisely fabricated 3D apparatus with painted patterns, such as two orthogonal planes hinged together, a cube, or a plane translated an exact amount in the normal direction. This makes sense since a camera records the projection of a 3D scene onto a $2 \mathrm{D}$ image plane, and if we know exactly what the $3 \mathrm{D}$ scene is, the camera parameters are readily determined. However, those 3D apparatuses are expensive to make and inconvenient to use. I wondered whether there could be a more flexible calibration technique.

With the knowledge of projective geometry I gained through my previous research career at INRIA in France, I discovered that we could calibrate a camera by just showing a planar pattern at a few different orientations (at least two) to the camera. The motion did not need to be known. Either the camera or the planar pattern could be freely moved, and the pattern could be printed on paper, then attached to a planar surface such as cardboard, making it truly flexible and easy to use.

This is rather counter-intuitive at first. We are only using a $2 \mathrm{D}$ object to calibrate a sensor which performs the projection from $3 \mathrm{D}$ to $2 \mathrm{D}$. Without going into the details, I will explain why this is possible. Algebraically, a 2D plane in 3D space and its projection in the image define a homography mapping which has eight parameters. The geometric relationship between the camera's 3D coordinate system and the plane's coordinate system has six degrees of freedom, which are unknown. Thus, each observation of a plane in space provides two constraints $(8-6=2)$ on the camera's internal parameters. We therefore need two (for a simplified camera model with rectangular pixels) or more (for a general camera model) observations of a plane in space.

My calibration technique can also be explained geometrically. This requires a concept called absolute conic in projective geometry. The absolute conic is a conic on a plane at infinity. Its projection on an image plane, irrespective of the camera position and orientation, remains the same and depends only on the camera's internal parameters. A plane in $3 \mathrm{D}$ space intersects with the absolute conic at a pair of conjugate points. Their projection in the image plane is also a pair of conjugate points and is determined by the homography matrix. With $N(N \geq 3)$ observations, we can determine the equation of the projection of the absolute conic, and in turn, the camera's internal parameters can be readily recovered from the conic equation.

Because of the ease of producing a planar pattern and the simplicity and robustness of the closed-form solution, my calibration technique is employed in computer vision research laboratories the world over, as well as by many companies. A version of this method calibrated the vision system in NASA's Mars Rover, while another version is used to recalibrate Kinect sensors to address the issue of sensor drift, saving 700 million dollars of potential product returns. In fact, it could well be the most widely used camera calibration algorithm extant.

This camera calibration technique was first described in an ICCV paper [5] and was later published in IEEE Transactions on Pattern Analysis and Machine Intelligence [3]. As of September 2017, these two papers have been cited more than 11,200 times, according to Google Scholar.

I developed the technology initially for my own need, and I did not have any idea that my work would have this sort of lasting impact. In 2013, I was rewarded with an IEEE Helmholtz Test of Time award. I am very happy that it has been used by so many people and companies. In retrospect, I did write in my paper, nearly two decades ago, that my algorithm "advances 3D computer vision one step, from laboratory environments to real-world use."

\section{References}

1. Liu, Z., Zhang, Z.: Face Geometry and Appearance Modeling. The Cambridge University Press, Cambridge, UK (2011)

2. Nishino, K., Zhang, Z., and Ikeuchi, K.: Determining reflectance parameters and illumination distribution from a sparse set of images 
for view-dependent image synthesis. In: International Conference on Computer Vision (ICCV). IEEE, pp. 599-606 (2001)

3. Zhang, Z.: A flexible new technique for camera calibration. IEEE Trans. Pattern Anal. Mach. Intell. (PAMI) 22(11), 1330-1334 (2000)

4. Zhang, Z.: Feature-based facial expression recognition: sensitivity analysis and experiments with a multi-layer perceptron. Int. J. Pattern Recognit. Artif. Intell. (IJPRAI) 13(6), 893-911 (1999a)

5. Zhang, Z.: 'Flexible camera calibration by viewing a plane from unknown orientations. In: International Conference on Computer Vision (ICCV). IEEE, pp.666-673 (1999b)

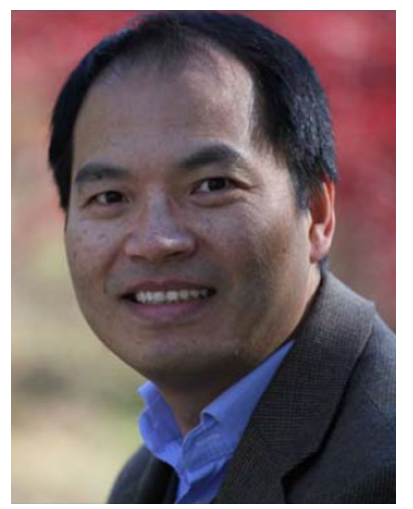

Zhengyou Zhang received the B.S. degree in Electronic Engineering from Zhejiang University, Hangzhou, China, in 1985, the M.S. degree in Computer Science from the University of Nancy, Nancy, France, in 1987, and the Ph.D. degree in Computer Science in 1990 and the Doctorate of Science (Habilitation à diriger des recherches) in 1994 from the University of Paris XI, Paris, France. He is a Principal Researcher with Microsoft Research, Redmond, WA, USA, and the Research Manager of the "Multimedia, Interaction, and Experiences" group. Before joining Microsoft Research in March 1998, he was a Senior Research Scientist with INRIA (French National Institute for Research in Computer Science and Control), France. In 1996-1997, he spent a one-year sabbatical as an Invited Researcher with the Advanced Telecommunications Research Institute International (ATR), Kyoto, Japan. He served as an Adjunct Chair Professor with Zhejiang University, Hangzhou, China. He is also an Affiliate Professor with the University of Washington, Seattle, WA, USA, and an Adjunct Professor with the University of Southern California, Los Angeles, CA, USA. He has published 250 papers in refereed international journals and conferences and has coauthored the following books: 3-D Dynamic Scene Analysis: A Stereo Based Approach (Springer-Verlag, 1992); Epipolar Geometry in Stereo, Motion and Object Recognition (Kluwer, 1996); Computer Vision (Chinese Academy of Sciences, 1998, 2003, in Chi- nese); Face Detection and Adaptation (Morgan and Claypool, 2010); and Face Geometry and Appearance Modeling (Cambridge University Press, 2011). He is an inventor of more than 120 issued patents and has received 8 Ship-It Awards from Microsoft. He has given a number of keynotes in international conferences and invited talks in universities. Dr. Zhang is a Fellow of the Institute of Electrical and Electronic Engineers (IEEE), a Fellow of Association for Computing Machinery (ACM), the Founding Editor-in-Chief of the IEEE Transactions on Autonomous Mental Development, an Associate Editor of IEEE Transactions on Circuits and Systems for Video Technology, and an Area Editor of the Journal of Computer Science and Technology. He served as Associate Editor of the IEEE Transactions on Pattern Analysis and Machine Intelligence from 2000 to 2004, an Associate Editor of the IEEE Transactions on Multimedia from 2004 to 2009, an Associate Editor of the International Journal of Computer Vision from 2004 to 2014, an Associate Editor of Machine Vision and Applications from 2004 to 2014, an Associate Editor of the International Journal of Pattern Recognition and Artificial Intelligence from 1997 to 2009, among others. He is on the Honorary Board of the International Journal of Computer Vision since 2015 and on the Steering Committee of the Machine Vision and Applications since 2015. He has been on the program committees for numerous international conferences in the areas of computer vision, audio and speech signal processing, multimedia, human-computer interaction, and autonomous mental development. $\mathrm{He}$ was a member of the Pre- and Interim Steering Committee, in 2009, in charge of revamping the International Conference of Multimedia and Expo (ICME), the flagship multimedia conference sponsored by four IEEE societies. He served as Area Chair, Program Chair, or General Chair of a number of international conferences, including recently a Program Co-Chair of the International Conference on Multimedia and Expo (ICME), July 2010, a Program Co-Chair of the ACM International Conference on Multimedia (ACM MM), October 2010, a Program CoChair of the ACM International Conference on Multimodal Interfaces (ICMI), November 2010, a General Co-Chair of the IEEE International Workshop on Multimedia Signal Processing (MMSP), October 2011 and September 2014, and a General Chair of International Conference on Multimodal Interaction (ICMI) 2015. He served as a Chair of a new track "Technical Briefs" of the ACM SIGGRAPH Asia Conference, November 28-December 1, 2012. He is serving as a General Chair of IEEE Conference on Computer Vision and Pattern Recognition (CVPR) 2017. He received the IEEE Helmholtz Test of Time Award at ICCV 2013 for his paper published in 1999 on camera calibration, now known as Zhang's method. 